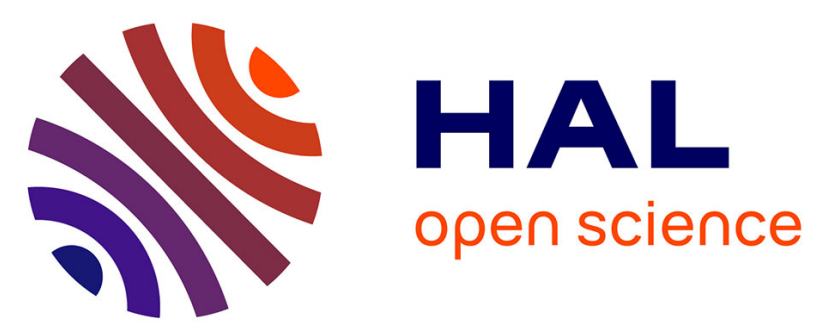

\title{
Un petit masque en tôle de bronze découvert à Genainville (Val-d'Oise) \\ Pierre-Henri Mitard
}

\section{To cite this version:}

Pierre-Henri Mitard. Un petit masque en tôle de bronze découvert à Genainville (Val-d'Oise). Gallia - Fouilles et monuments archéologiques en France métropolitaine, 1982, 40 (2), pp.287-291. 10.3406/galia.1982.1867 . hal-01934199

\section{HAL Id: hal-01934199 \\ https://hal.science/hal-01934199}

Submitted on 27 Feb 2020

HAL is a multi-disciplinary open access archive for the deposit and dissemination of scientific research documents, whether they are published or not. The documents may come from teaching and research institutions in France or abroad, or from public or private research centers.
L'archive ouverte pluridisciplinaire HAL, est destinée au dépôt et à la diffusion de documents scientifiques de niveau recherche, publiés ou non, émanant des établissements d'enseignement et de recherche français ou étrangers, des laboratoires publics ou privés.

\section{(ㅇ)(1) $\$$}

Distributed under a Creative Commons Attribution - NonCommercial - NoDerivatives| 4.0 


\title{
UN PETIT MASQUE EN TÔLE DE BRONZE DÉCOUVERT À GENAINVILLE (Val-d'Oise)
}

\author{
par Pierre-Henri MITARD
}

\begin{abstract}
Alors que les sculptures antiques en toile de bronze, que l'on considère généralement comme l'expression d'une tradition celtique, sont relativement rares, le site gallo-romain des Vaux de la Cielle à Genainville en a livré, pour sa part, trois, de types et de tailles divers. La plus importante, une tête humaine grandeur nature, a fait l'objet récemment d'une élude détaillée dans les pages mèmes de celte revue ${ }^{1}$, a l'occasion de laquelle l'une des deux autres: partie supérieure d'un masque un peu plus petit que nature. a également été présentée ${ }^{2}$. C'est it la troisieme, de plus petite taille et de fonction vraisemblablement différente. qu'est consacrée la présente note.

Il s'agit d'un petit masque découvert en 1978 , a 6 m au nord de l'angle n.-e. du temple (en ‥ 20,75)- E. 17,30), a une cinquantaine de centimitres sous le niveau du sol de service de ce monument. Le lieu de trouvaille se situe tout près de l'emplacement du bàtiment secondaire VIII, dans les vestiges duquel la tète précitée a été recueillie. mais la couche
\end{abstract}

X.B. - Qu'il nous soit permis d'associer à ce travail notre collegue de fouille B. Viollat, fouilleur assidu de ce secteur, inventeur de ce petit masque. Origine des documents: fig. 2 , photo Nationalmuseet, Copenhague; fig. 3 et 4, dapries sir 6. Fox, Pattern and Purpose.

1 P.-H. MitaRI, La lite en tôle de bronze de (ienainuille (Val dOCise), dans Ciallia 40, 1982, p. 1-33. ann'xes.

2 Op. cil. annexe III. dans laquelle se trourait ce masque est en fait antérieure à l'érection des deux monuments.

Ce masque, conservé au musée de Guiry-enVexin (inv. G. 1552), est en tòle de bronze d'environ $4 / 10$ de $\mathrm{mm}$ d'épaisseur, portant des traces d'étamage. Sa largeur maximale est de $0,05 \mathrm{~m}$, pour un relief maximal de $0.02 \mathrm{~m}$. Il est incomplet à la périphérie, en particulier a partir du niveau du front (tig. 1). Le bas du visage est entouré d'une sorte de rollerelle cotelée paraissant se poursuivre a l'origine sur les còtés (d'apriss des restes du còté gauche). L.es yeux, figurés par un tracé ovale allongé en pointes légèrement en creux, avec un trou rond a l'emplacement de la prunelle (pupille-iris), étaient surmontés d'arcades sourcilieres marquées. malheureusement en grande parlie détruites par l'oxydation. Le menton et la bouche sont figurés par des reliefs doux ; un petit relief peut correspondre au départ de l'oreille gauche. Le nez rectiligne, mais incurvé à l'extrémité, est en saillie de $7 \mathrm{~mm}$.

Les recherches effectuées à l'occasion de l'étude de la têle de bronze évoquée ci-dessus nous ont amené à constater que ce petit masque pouvait ètre rapproché, tant par sa technique et son style que par sa taille. de masques en métal battu et repoussé appartenant à la décoration des parois de plusieurs grands récipients de caractère rituel ou mème de replle de la caisse d'un rhar. Ces pieces de 


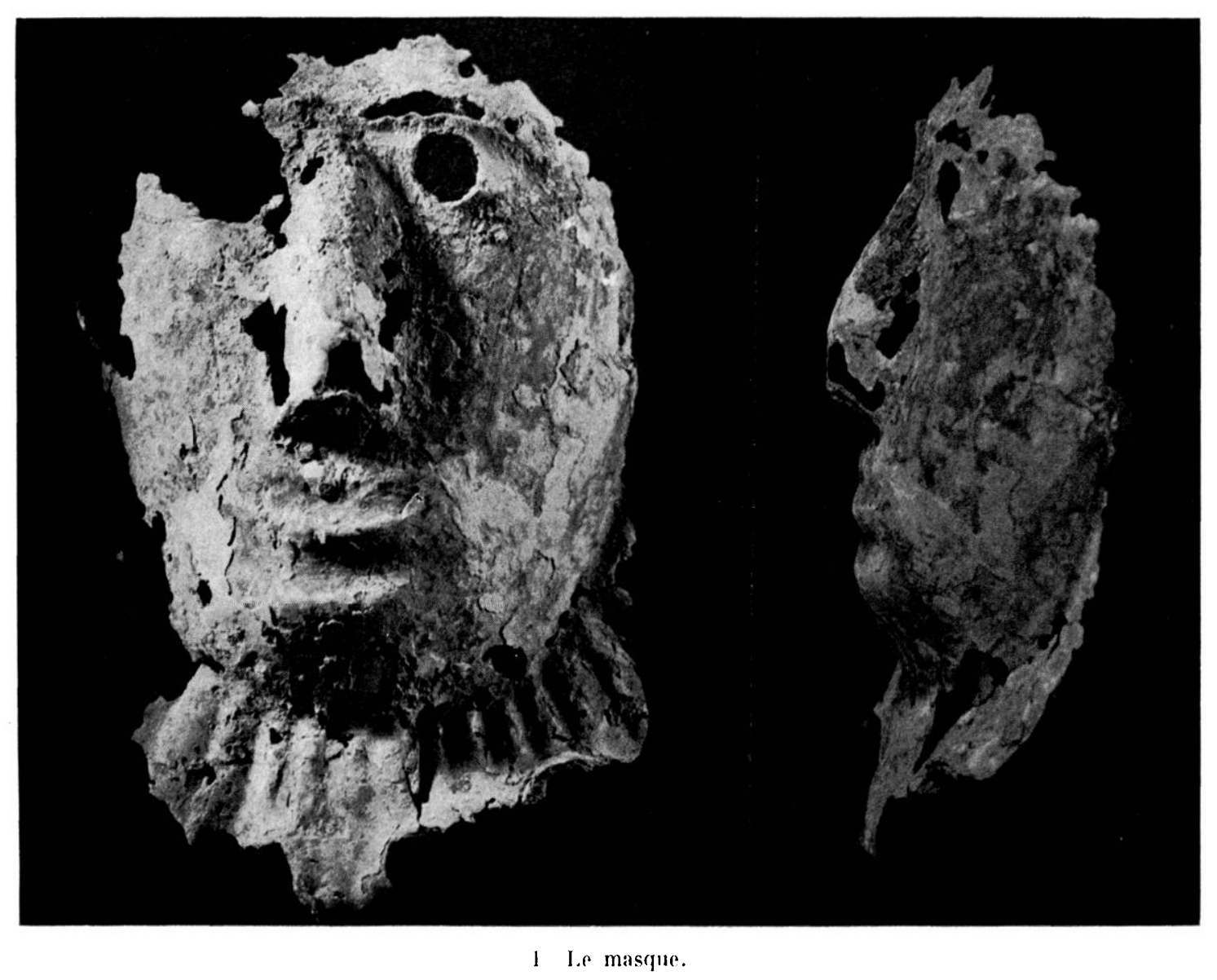

romparaison, datables du ter sibcle as. J.-C. jusqu'aux environs du ${ }_{1}^{\text {er }}$ siécle de notre ère. onl été réunies dans une série de notices données ci-apris et auxquelles renvoient des numéros entre crochets. Les masques en question présentent une schématisation des traits qui se relrouve sur celui de Genainville. mais aussi. pour la plupart. une stylisation typiquement celtique. Certains dentre eux figurent sur un document insigne : le bassin en argenl doré de Gundestrup "1]. L'un des éléments caractéristiques est le mode de figuration des yeux : un ovale allongr en pointes, percé d'un trou rond a l'emplacement de la prunelle, que présentent en commun ces diverses pieces, avec des variantes de détail. Sur le bassin de Gundestrup, les trous ronds de certains yeux portent encore des pastilles de verre de couleur rouge ou bleue : on peut penser qu'il pouvait en ètre de mème. pour les autres masques ef en particulier pour relui de Genainville.

Quant aux dimensions, elles sont du mèrne ordre de grandeur pour ces diverses pirces; compte lenu de leur etat de conservation, r'est la largeur du visage qui permet le plus aisément la comparaison, elle est d'environ $0.08 \mathrm{~m}$ pour les masques du bassin de Gundestrup 1; 0,09 $\mathrm{m}$ pour celui de sophienborg 2 ; $0,09 \mathrm{~m}$ pour le mieux conservé des masques du seau de Marlborough [3]; un peu moins de $0,06 \mathrm{~m}$ pour les masques du char de stanwick 4 . I a largeur de celui de Genainville est un peu plus faible (0.05) $\mathrm{m}_{j}$.

Peut-on, comple tenu de ces exemples. imaginer que ce petit masque aurait à l'origine orné, avec d'autres éléments disparus, la paroi d'un bassin ou d'un seau. ou éventuellement. d'un véhicule. comparable à ceux qui viennent 
d'être cités? Cela ne nous parait pas impossible, mais ne constitue qu'une possibilité, puisque la pièce n'est pas suffisamment bien conservée pour offrir, par quelque détail de la périphérie par exemple, un élément supplémentaire d'appréciation, et que la fouille n'a pas jusqu irci livé d'autres fragments susceptibles de provenir d'une piece de ce genre.

Quant a la datation, te contexte de découverte en fournit des éléments d'appréciation : il s'agit d'une couche tris humide paraissint correspondre au rolmatage d'une fonlaine la source initiale qui est a l'origine de la fréquentation du site noetait certainement pas loin, recouverte vers le milieu du ne sierle d'une couche de remblai, et dans laquelle ont ité recueillies ... sur un espace d'a peine 5) $\mathrm{m}^{2}$. 45) monnaies de bronze (as) s'echelonnant d'Auguste a kaustine Mire Diva, la derniere (B.M.C. 1590), dalable d'un peu apris 141. Le masque, trousí au niveau le plus profond et qui n'aurait pu s'y enfoncer par son propre poids, a dù ètre déposé aver les monnaies les plus anciennes: mais sa date de fabrication peut évidemment itre un peu antérieure et rejoindre ainsi sans difficullé celle des éléments de comparaison précédemment inentionnés, dont les datations se siluent entre le début du i ${ }^{\text {er }}$ siòcle av. et le milieu du $1^{\text {er }}$ siecle ap. J.-C.

\section{PIBCEN DE: (OOMPARASON}

1. Cindeslrup, Raevemosen, Lars, Jutland (1)anemark), dans une tourbicre, 1891. Vationalmuseet, Copenhague.

"Chaudron" ou plutot bassin, en argent doré. comportant, a l'extérieur, des plaques ornees de bustes de divinites (4 masculines, :) féminines) entourées d'autres motifs. Haut. des teites: environ $0,10 \mathrm{~m}$, pour $0,08 \mathrm{~m}$ de largeur. Sur ces têles, exécutées dans les plaques au repoussé comme le reste du décor. les yeux sont tigurés par des reliefs de forme ovale aver pointes. ou en amande, perces a l'emplacement de la prunelle de trous ronds, dans lesquels etaient fixés a la résine, semble-t-il des pastilles de verre de

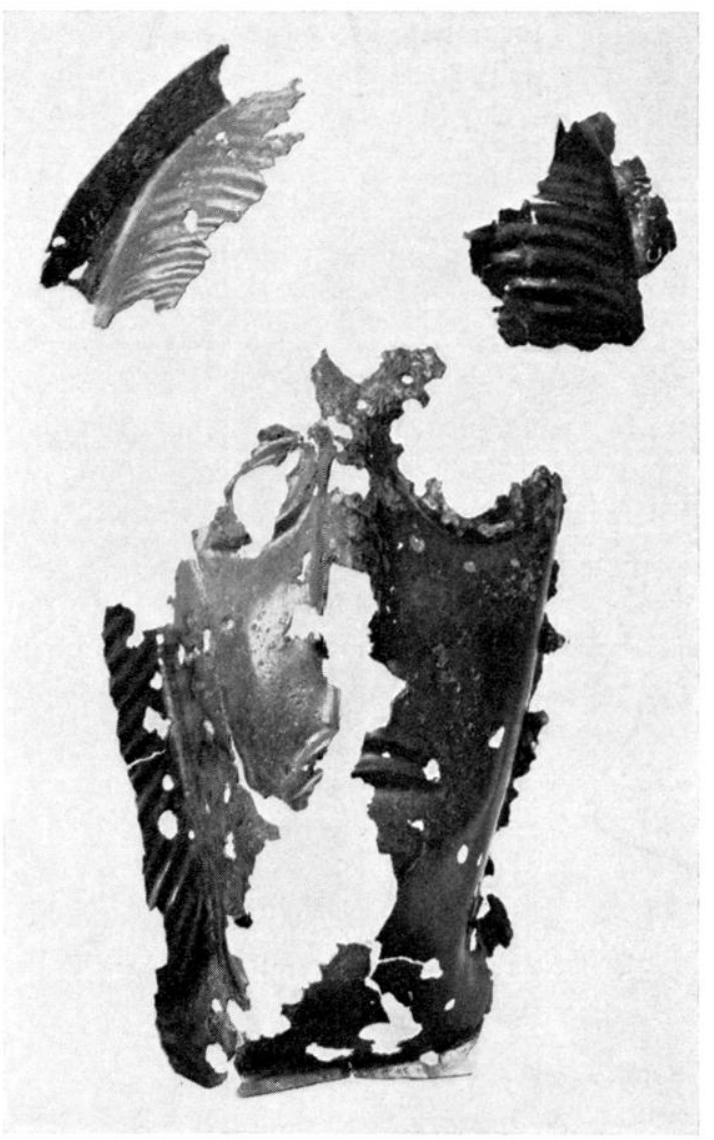

$\because \quad$ Masciue de Sophienborer.

rouleur rouge on blene (quatre de celles-ci subsistent, sur 3 bustes différents : b, e, e). Lieu de fabrication discute : Gaule ou région du Pont-liuxin? Tatation probable : ${ }^{\text {er }}$ s. av. J.-C.

S. Muller, 1892. O. Klindt-Jensen, 1949, 1. 119 et s. particuliirement fig. 77. A. Varagnac, 1935, p. 2:36, nos 3 a 10. F. Benoit, 1969, ill. 5, fi7 a 73. J. V. s. Hegaw, 1970, no 209. J.-J. Hatt, 1970. p. 103, fig. 51-60. P.--Y. J)uval, 1977, p. 18.188. G. s. Olmsted, 1979, particuliirement pl. 4. 5 .

2. Sophienborg, priss de Itillerod $(30 \mathrm{~km}$ au n.-e. de Copenhague, lanemark), dans un marais. Nalionalmuseet, Copenhague.

Petil masque féminin?) aver cou, en plusieurs fragments et incomplet. Tóle de bronze. Largeur maximale du visage : $0,09 \mathrm{~m}$ env:; relief maximal : 0,04 in évaluation d'apres les illustrations publiées, figr. 2). La 
chevelure, formée de mèches régulières exécutées au repoussé de l'inlérieur, encadrait le visage jusqu'au niveau du menton. les yeux. très obliques, sont figurés par un tracé ovale allongé en pointes, en relief, avec un lrou rond a l'emplacement de la prunelle. Le menLon el la bouche sont, bien marqués en relief.

Trouvé avec des fragments d'un chaudron et de têtes de taureau en fonte de bronze, analogues a ceux ornant un autre chaudron ou bassin mieux conservé découvert. également au Tanemark, à Rynkeby, ce masque devait avoir la mème fonction que celui en fonte de bronze ornanl la paroi de ce dernier. De profil, l'arrière de la piece. apparaît d'ailleurs fait pour s'ajuster à la courbure de la paroi d'un tel récipient. I.es pieces de sophienborg (comme relle de Rynkeby) sont considérées comme d'origine gauloise et datables du ${ }_{1}^{\text {er }}$ s. av. J.-C.

II. Pelersen, 1888, p. 39. S. Muller. 1892, p. 59, figr. 11 et 12. (). Kilindt-Jensen. 1949. p. 116, lig. 69 a.
3. Warlhorough. Wiltshire (Angleterre), sépullure a incincration (?) 1807. Tevizes Museum.

Fragments d'éléments de décoration en tòle de bronze appartenant a un seau i douves en bois avec cerclages de fer; parmi res aléments. une bande ornée - a des emplarements non contigus de deux masques humains de face en haul-relief fel de molif: animaliers diversi. Iaro. d'un masque : $0,09 \mathrm{~m}$ env. (evaluation) (fig. 3). La figuralion de la chevelure de l'un des masques est asso\% élaborée. les yeux, au contour ovale allongé en pointe en relief, presentent un trou rond a l'emplacement pupille-iris. Origine gauloise.

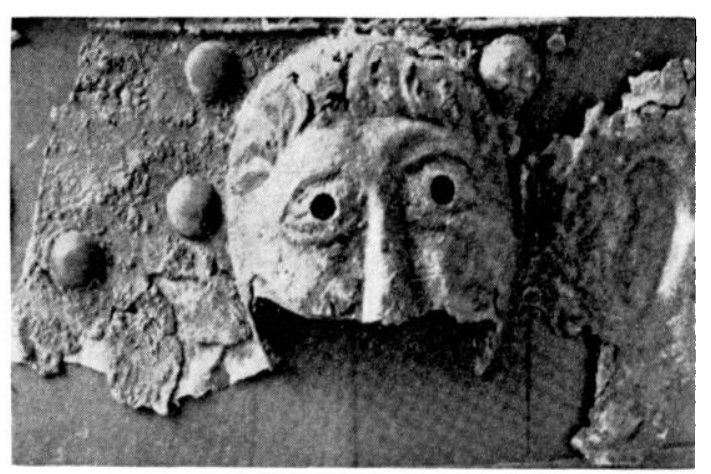

3 Maspgur shr seatu de Marlborough.

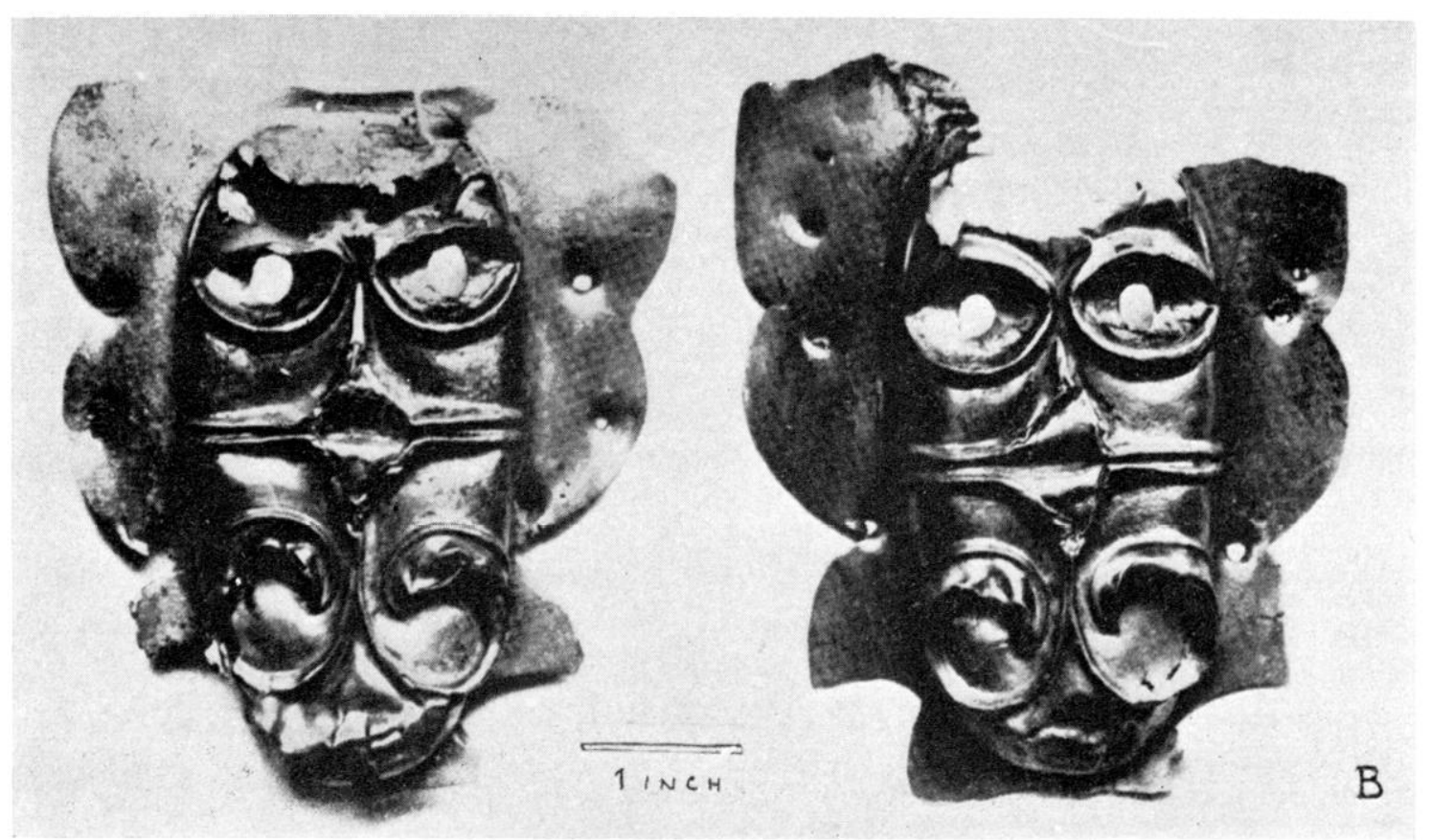

1 Masque sur caisse de char,?, de Stanwick. 


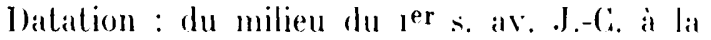
pre moilie du jer s.

E. Aylen, 1958, fig. 6.7. C. Fox, 1958. p. 68. pl. 34-36. X. K. Sandars, 1968, fig. 297300. J. V. s. Megaw, 1970, p. 120, no 180. (i. s. Olmsted. 1979, p. 49-5)3, pl. 2(i-29.

1. Stanuick, Yorkshire (Angleterre). Arant 1846. British Museum.

Reliefs ornant la caisse d'un char (?) fou une maison de hois?). Haul. : $10 \mathrm{~cm}$ (fịt. 1 ). Deux masques ovales aver yeux présentant un trou rond a l'emplacement de la prunelle.

"Visage " séparé en deux dans le sens vertical par un double relief horizontal formant axe de symétrie; la moilié inférieure est oreupée par deux volutes en spirale de sens opposes. symétriques des yeux; composition monstrueuse lypiquement celtique. Datalion : I $^{\text {er }} \therefore$.

C. Fox. 1958. p. 73. pl. 43 b. J. V. S. Megaw, $197\left(1 . n^{\circ} 2(6)\right.$.

N.B. - Des pelits masques (haut. : 6 cm) d'un style proche des nos 2 et 3 précédents (veux figures de la même maniere) ornent la raisse d'un chariot rituel découvert a Tejbjeror (Jutland. Danemark) (II. Platensex, 1888, pl. I). Jous ne savons pas s’ils sont en tòle ou en fonle de bronze.

\section{Pierre-Henri Mitani)}

(G.N.R.S.)

\section{BHBLIOGRAPHIF}

Fernand Brosotr. Art et Dieux de la tiaule, 1969 .

Paul-Marie Deval. Les Celles, coll. L'Lnivers des Formes, Paris, 1977.

Jan Funp. Die Kellische Zinilisation und ihr Erbe, Prague, 1961.

Civil fox Sir. Paltern and Purpose. A Survey of Early Cellic art in Brilain, Cardiff, 195x.

Jean-Jacyuts Hatr. Celles el gallo-romains, coll. Archaclogia Mundi, 1970.

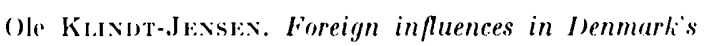
Early Iron Age, dans Acla Archaelogica, vol. XX. copenhague, 1949

John Vincent Stanley Mrasw. Art of the European Iron Age, Bath, 1970.
Sophus Mindik. Del store sollivar fra (iundestrup $i$ Jylland, dans Nordiske Forlidsminder 1, p. 36$3 \times, 1 \times 92$.

Erik Nyis. The remarliahle bucliel from Marlborough, dans Acla Archaenlngica, XXIX, Copenhague, p. $1-20,1958$.

Garmel S. Ornsten. The Ginndestrup cauldron, coll. I.alomus, vol. 162, Bruxilles, 1979.

Henry Petersas. Lognfundene $i$ hejbjerg Iraeslegatardsmose red kinglijobing 1881 og 188.3, Copenhague, $1 \times \times \times$.

Nancy Katherine SAxusks. Prehisloric Arl in Europe, The Pelican history of art, Hermondsworth, 1968.

Imbri Varagxac: cel Gabrielle Fabre . L'arl gaulois, coll. Ia Nuit des Temps, 1956 . 